\title{
MULTI-AREA QOS PROVISIONING USING HIERARCHICAL BANDWIDTH BROKERS ARCHITECTURE
}

\author{
Ling Teck Chaw, Phang Keat Keong, Ang Tan Fong, Lim Gek Pei \\ Faculty of Computer Science and Information Technology, \\ University of Malaya, Kuala Lumpur, Malaysia \\ Email: \{tchaw, kkphang, angtf\}@um.edu.my; gek_pei@hotmail.com
}

\begin{abstract}
In this paper, an intra-domain, multi-area Quality of Service (QoS) provisioning mechanism, namely the QoS-HBB architecture is proposed to alleviate the scalability problem in the intra-domain bandwidth broker design. By exploiting the inherent hierarchical nature of OSPF and the fact that an OSPF domain can be subdivided into multiple areas, QoS-HBB introduces two levels (i.e. intra-area and inter-area) of QoS provisioning within a domain. The proposed architecture allows the deployment of multiple bandwidth brokers in the backbone area and non-backbone areas. At the intra-area level, a child bandwidth broker is deployed in every non-backbone area to handle intra-area QoS provisioning; whereas, at the inter-area level, QoS control functions are delegated from the child bandwidth broker to the parent bandwidth broker situated at the backbone area. With the hierarchical design and efficient QoS state information maintenance, QoS-HBB is capable of providing service assurance to end-user applications. Furthermore, Multi-Protocol Label Switching (MPLS) and path caching are incorporated to improve the performance. Simulation results show that, compared to ordinary OSPF, QoS-HBB significantly improves QoS provisioning in terms of packet loss ratio, link utilization, end-to-end delay, packet commit percentage, and throughput.
\end{abstract}

\section{Keywords: QoS, Bandwidth Broker, OSPF, MPLS, Hierarchical Network}

\subsection{INTRODUCTION}

Today, as the Internet evolves into the ubiquitous communication networks, it has become an essential communication channel across the globe. This is evident by the increased data and multimedia traffic such as video conferencing, video-on-demand, IP telephony, video and audio streaming, and critical business transactions. However, the traditional Internet which is best-effort in nature is unable to cope with this change as it does not support Quality of Service (QoS) provisioning such as resource reservation and data delivery with assured quality. As a result, numerous mechanisms such as constraint-based routing, Integrated Services (IntServ), Differentiated Services (DiffServ), Multiprotocol Label Switching (MPLS), Resource Reservation Protocol (RSVP), traffic engineering and bandwidth broker have been proposed to provide some form of QoS support in the IP network [1, $2,3,4,5,6,7]$.

At the routing level, mechanisms such as QoS routing is suggested to overcome existing limitations by finding feasible network paths that meet the given constraints and then reserve the resources in order to support and guarantee the specific service $[8,9,10,11]$. However, major challenges which exist in constraint-based routing include scalability, robustness, and stability [12]. Scalability is contributed by computational cost and protocol overhead. Computational cost refers to the cost of path selection computations whereas the protocol overhead is the overhead of distributing state updates. Robustness is related to the inaccuracy of the available state information that could affect the performance of QoS routing. Stability concerns the amount of state information maintained at every node and also the frequency of link state updates.

An effort which parallels the effort of constraint based routing is the extension of the Open Shortest Path First (OSPF) routing protocol to support QoS. OSPF is a link state routing protocol developed by the OSPF working group of the Internet Engineering Task Force (IETF) for deployment in the TCP/IP internet environment [13]. As an Interior Gateway Protocol (IGP), OSPF controls the routing within a single Autonomous System (AS). In order to increase the network scalability, OSPF divides an AS into areas and constructs hierarchical routing among the areas. OSPF supports two-level hierarchy areas within the AS. The top level hierarchy is known as the backbone area or Area 0 which provides connectivity to the rest of areas at the second level. The backbone area is responsible for distributing routing information to and from the non-backbone areas. QOSPF is an extension of OSPF which allows 
the inclusion of network resource information such as available bandwidth in the link state advertisement. It also supports path computation based on resource information. [14].

Another major effort was in the direction of bandwidth broker which is the focus of this paper. Within the IETF Differentiated Service framework [15], the concept of bandwidth brokers is defined as an entity which can be configured with organizational policies, keeping track of the current allocation of marked traffic, and interpreting new requests to mark traffic in light of the policies and current allocation. Bandwidth brokers allow the automatic provisioning and management of a network through pre-agreed policy and arrangement. In this paper, we propose and design a hierarchical BB framework using QOSPF to address the issue of resource allocation and management in an intra-domain basis. In particular, the framework is applied in an AS which runs multi-area OSPF. QoS provisioning within a non-backbone area is managed by the local Child Bandwidth Broker (CBB), whereas for QoS requests destined to other non-backbone area, the QoS provisioning is managed by the Parent Bandwidth Broker (PBB).

The paper is organized as follows. Section 2 presents literature survey and related works. Section 3 discusses the proposed mechanism, while Section 4 presents the simulation results and the performance analysis. Finally, Section 5 concludes the paper with future work.

\subsection{LITERATURE REVIEW}

Bandwidth brokers are a complex entity involving the integration of technologies such as inter and intra domain communication (such as Border Gateway Protocol, OSPF), standard protocols (such as DiffServ, IntServ, MPLS) and database. An intra-domain bandwidth broker, as the name implies, works within a domain. Its responsibility includes configuration of edge routers within the domain to enforce resource allocation and admission control. An inter-domain bandwidth broker, on the other hand, is responsible for QoS parameters negotiation and the setting up of bilateral agreements among the bandwidth brokers at the neighbouring domains.

The work by Nichols et al. [15] is an IETF initiative to propose a centralized bandwidth broker for the control and management of QoS within a domain. This centralized bandwidth broker support admission control, resource reservation and QoS provisioning for the entire domain. This model has the advantage of separating the QoS control plane from the data plane. Admission control and QoS state maintenance are removed from the routers and handled by the bandwidth broker to reduce the work load of the routers. In contrast to the IETF IntServ QoS control model, this approach uses the DiffServ approach. Here, QoS flows are handled in an aggregated manner based on the DSCP in the packet header instead of the per flow basis as in the IntServ approach. Hence, the centralized bandwidth broker model provides a more efficient alternative for QoS control and management.

Zhang et al. proposed an intra-domain, centralized, path-oriented, quota-base bandwidth broker [16]. The objective of the research is to study the scalability issues of the centralized bandwidth broker model for flow-level dynamic QoS control and management. The proposed bandwidth broker is later extended to hierarchically distributed architecture with the deployment of multiple bandwidth brokers within a single domain. The proposed hierarchical model consists of edge bandwidth brokers and a central bandwidth broker. The edge bandwidth brokers manage a mutually exclusive subset of path QoS states and admission control while the central bandwidth broker maintains the link QoS state database and manages quota allocation.

In addition, Gadgil et al. proposed an intra-domain bandwidth broker to achieve QoS assurance with fault tolerance support [17]. The proposed bandwidth broker uses OSPF in its route discovery. The bandwidth broker obtains the OSPF link-state information from routers adjacent to a fault and performs a QoS impact analysis for various flows. Lee et al. presented an 'emulation environment' for the design and planning of QoS-enabled IP networks with bandwidth broker [18]. Each router is able to compute multiple QoS paths in parallel to the same destination to improve network reliability. Vivanco and Jayasumana dealt with single domain bandwidth broker that supports QoS at an aggregate level using Service Level Agreements (SLA's), and the QoS Routing proposed in this paper was built on top on the OSPF routing protocol [19]. Each router not only stores the OSPF route database but also stores alternate paths to other routers based on QoS parameters such as delay bound and bandwidth. Note that the proposed intra-domain bandwidth brokers in all the above papers [15, 17, 18, 19] are restricted to one bandwidth broker per domain and hence do not scale well. 
Spence proposed an inter-domain bandwidth broker [20]. The research focuses on the role of BB in authorizing and the establishment of services similar to the expedited forwarding (EF) per hop behavior (PHB) in multi-domain scenario. Likewise, an inter-domain bandwidth broker was also proposed by Bouras and Primpas [21]. Relevant aspect of the operation of inter-domain BBs was discussed with the focus on path finding performed by the interdomain bandwidth brokers. Two path finding mechanisms, namely the centralized and the peer to peer models were described. A framework of two-tier intra-domain and inter-domain bandwidth brokers is attributed to Terzis et al. [22]. Individual administrative domains are able to independently make their own decisions on strategies and protocols to be used for internal resource management and QoS support. A simplified version of RSVP is used as an intra-domain resource allocation protocol to aggregate traffic between border routers. In this model, a single bandwidth broker controls the entire domain.

To the best of our knowledge, all previous works on intra-domain bandwidth brokers except [16] concern the deployment of a single bandwidth broker for the entire domain. This architecture imposes scalability issues. For intra-domain bandwidth brokers, the number of routers within a single domain depends on the types of area, CPU power, media used, etc. There can be as large as a few hundred routers within a single area. Factors affecting scalability in the design of intra-domain bandwidth broker include admission control, accessing and updating of QoS state, and the capacity of the communication channels between the bandwidth broker and the routers. The processing burden on the only bandwidth broker within an area will be extremely high; this results in both the communication channel to the bandwidth broker and the bandwidth broker to become the bottleneck. In other words, current proposed intra-domain bandwidth brokers do not scale well in network with large number of routers and high volume of traffic. Therefore, there is a need to develop hierarchical intra-domain bandwidth broker architecture to address this problem.

In [17, 21, 22], the research are restricted to one bandwidth broker per domain and hence do not scale well. In order to improve scalability, our proposed QoS-HBB, on the other hand, supports more than one bandwidth broker per domain. Also, while both [16] and QoS-HBB support multiple bandwidth brokers per domain, [16] lacks the following crucial details: (i) databases of the bandwidth broker, (ii) how the central bandwidth broker communicates with edge bandwidth brokers, and (iii) how the bandwidth brokers obtain QoS information about a link or a path from the communication protocols. These details are defined and included in the QoS-HBB proposal.

\subsection{PROPOSED MULTI-AREA INTRA-DOMAIN QOS MECHANISM — QOS-HBB}

Our work focuses on intra-domain bandwidth brokers. Specifically, a hierarchical bandwidth broker, namely QoSHBB is proposed. QoS-HBB obtains the OSPF link-state information from the routers to perform the QoS provisioning task. In this design, multiple bandwidth brokers can be deployed within a domain. Each OSPF area contains a bandwidth broker to manage the network resources and determines the QoS paths on behalf of all routers within its controlled area. The bandwidth broker is located in or close to the middle of the area in order to shorten the average distance between the bandwidth broker and the routers. The bandwidth broker located in the backbone area is called the Parent Bandwidth Broker (PBB) and the bandwidth brokers located in the non-backbone areas are called Child Bandwidth Brokers (CBBs). Compared to a CBB, PBB is more powerful as it manages both intra-area QoS routing for the backbone and inter-area QoS routing. The architecture of QoS-HBB is illustrated in Fig. 1. In Fig. 1, BB1 and BB2 are CBBs. For the rest of the sections in this paper, all discussion is based on the architecture shown unless otherwise stated. 


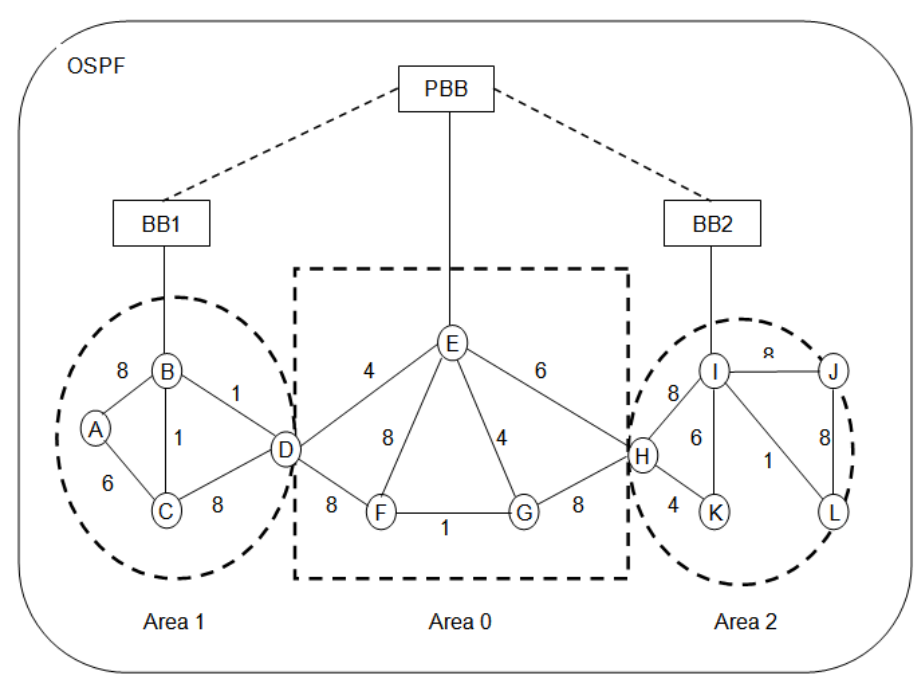

Fig. 1: The QoS-HBB Architecture

Table 1: Link QoS State Database of CBB1

\begin{tabular}{|l|l|l|l|l|}
\hline & A & B & C & D \\
\hline A & - & 8 & 6 & - \\
\hline B & 8 & - & 1 & 1 \\
\hline C & 6 & 1 & - & 8 \\
\hline D & - & 1 & 8 & - \\
\hline
\end{tabular}

Here are the assumptions made for the proposed mechanism:

1. The routers only delegate the QoS flows routing decision to PBB and CBBs. The best effort flow is handled by the routers using the existing OSPF routing table.

2. In the network, either a certain amount of network resource is reserved for QoS traffic, or the QoS traffic has higher priority than best effort traffic and thus can preempt the resource of best effort traffic. Under this assumption, the PBB and CBBs are always able to maintain the accurate link QoS state information.

3. The routers in the domain support DiffServ and MPLS.

\subsection{Network Topology Maintenance}

Before PBB and CBBs are able to calculate the QoS paths on behalf of the routers, they must have complete and upto-date network topology information. As QoS-HBB is running in an OSPF domain, the PBB and CBBs can passively involve in OSPF routing by listening to the LSAs flooded throughout the network to gather the network link state and topology information. The implementation of the passive listener is simple and straight forward and does not incur additional overhead even when the network is large. In this snooping mode, a CBB listen to all the Router-LSAs in the area under its controlled. The CBB then sends and receives Router-LSAs to PBB. By combining the LSAs from the backbone area and the LSAs from all CBBs, PBB acquires full knowledge of the entire domain or AS topology.

\subsection{Data Structures}

In QoS-HBB, each bandwidth broker maintains two set of tables for the purposes of QoS control and resource management of the network:

1. Link QoS State Database (LSDB)

LSDB is built using the network topology information gathered through the snooping mechanism mentioned earlier. This table not only includes the network topology information but also the current QoS state of every link in the network. The LSDB is a $K \times K$ matrix and $K$ is the number of nodes in a domain 
or the network. The matrix value for $(s, d)$ entry denotes the available bandwidth of the link connecting node $s$ to node $d$. If link does not exist from node $s$ to node $d$, the entry is left blank.

The size of the PBB's LSDB is larger compared to the CBB's LSDB. This is because the PBB's LSDB covers all the links in the entire OSPF domain, whereas the CBB's LSDB only includes the links within the area controlled by the CBB. Table 1 and 2 are examples of PBB's and CBB's LSDBs. Note that in the beginning, before any path request is received, the available bandwidth of a network link equals to its static link capacity.

\section{QoS Path Cache (QPC)}

QPC is a table that stores the QoS paths calculated from the previous requests. The purpose of the cache is to reduce the computational overhead of the PBB and CBBs. For any subsequent QoS path request involving the same pair of source and destination, $\mathrm{PBB}$ and $\mathrm{CBBs}$ can directly respond to the request by referring to directly the path stored in the table without path recomputation if the stored path satisfies the request. In order to reduce the size of the cache, only information of the source, destination and path are stored. Hop count and other QoS parameters are excluded. Furthermore, for every destination-source pair, only one path is stored. An example of the QPC of CBB1 is shown in Table 3.

Table 2: Link QoS State Database (LSDB) of PBB

\begin{tabular}{|l|l|l|l|l|l|l|l|l|l|l|l|l|}
\hline & A & B & C & D & E & F & G & H & I & J & K & L \\
\hline A & - & 8 & 6 & - & - & - & - & - & - & - & - & - \\
\hline B & 8 & - & 1 & 1 & - & - & - & - & - & - & - & - \\
\hline C & 6 & 1 & - & 8 & - & - & - & - & - & - & - & - \\
\hline D & - & 1 & 8 & - & 4 & 8 & - & - & - & - & - & - \\
\hline E & - & - & - & 4 & - & 8 & 4 & 6 & - & - & - & - \\
\hline F & - & - & - & 8 & 8 & - & 1 & - & - & - & - & - \\
\hline G & - & - & - & - & 4 & 1 & - & 8 & - & - & - & - \\
\hline H & - & - & - & - & 6 & - & 8 & - & 8 & - & 4 & - \\
\hline I & - & - & - & - & - & - & - & 8 & - & 8 & 6 & 1 \\
\hline J & - & - & - & - & - & - & - & - & 8 & - & - & 8 \\
\hline K & - & - & - & - & - & - & - & 4 & 6 & - & - & - \\
\hline L & - & - & - & - & - & - & - & - & 1 & 8 & - & - \\
\hline
\end{tabular}

Table 3: Example of QoS Path Cache (QPC) maintained by CBB1

\begin{tabular}{|l|l|l|}
\hline Source & Destination & Path \\
\hline A & B & A, B \\
\hline A & C & A, C \\
\hline A & D & A, C, D \\
\hline B & A & B, A \\
\hline C & B & C, B \\
\hline D & C & D, C \\
\hline
\end{tabular}

\subsection{Path Computation}

Without path caching, path computation is invoked whenever the bandwidth broker receives a path request. With path caching, previous on-demand calculated paths are stored in the database for future reference. Therefore, path computation is only triggered if there is no entry in the cache that can satisfy the request. QoS-HBB uses extended Dijkstra shortest path algorithm in path finding. The traditional Dijkstra shortest path algorithm is modified to include both the hop-count and the available bandwidth in the path computation metric. The widest-shortest path heuristic [23] is used as the basis of the path computation algorithm. The goal of the modified Dijkstra's algorithm is to find a path with the minimum number of hop count that can support the requested bandwidth and also has maximum bandwidth. The algorithm first prunes all the links that do not have the required available bandwidth. Then based on the pruned graph, the minimum hop count path between source and destination is determined. If more than one path that satisfies the path request is available, the widest one (with the highest available bandwidth) is selected. 


\subsection{Operation Details}

QoS-HBB operates in the following manner:

1. When a QoS path request with source $s$, destination $d$, and bandwidth requirement $b w$ arrives at a router of the non-backbone area, the router generates and sends a Resource Allocation Request (RAR) to the CBB in its area. For a router resides in the backbone area, the RAR is sent to the PBB instead.

2. When a CBB receives the RAR, it checks whether the destination node $d$ is within its controlled area. This process is done by examine the Router-LSAs maintained by CBB to see whether there is a link with link type 'Link to stub network' and the link ID matches with $d$. If the link exists, this means that the destination is within the CBB's controlled area. This is the case for intra-area; otherwise it will be an interarea case.

\section{Intra-area Case:}

If the CBB detects that the destination address of a request is within its controlled area, the operation proceeds as follows:

3. CBB looks up QPC for the entry with source field equals to $s$ and destination field equals to $d$. If the entry is found, the QoS path of that entry is retrieved. Then based on the LSDB, the CBB checks whether each link constituting the path is able to satisfy the request, that is, the available bandwidth of each link is greater than or equal to the requested bandwidth, $b w$.

4. If the path retrieved satisfies the request, CBB simply retrieves the path information without re-calculating a new one. For the case in which either there is no such entry in the QPC or the path found in the entry fails to support the request, CBB performs the on-demand Dijkstra algorithm to calculate a feasible path for the request, based on the topology and network state information stored in its LSDB.

5. When the feasible path for the request is found, CBB updates the path information in the QPC (by adding a new entry if one does not exist, or by replacing the path of current entry with the new path information). At the same time, CBB updates its LSDB. For every link of the feasible path, its available bandwidth is reduced by $b w$ to reflect the resource allocation to the request. Later, $C B B$ sends an update link state message to PBB such that PBB is able to update its LSDB for the corresponding entry.

6. CBB generates a Resource Allocation Answer (RAA) and sends it to the requesting router. The RAA indicates whether the request is admitted or rejected. For a positive reply, the path information is inserted into the RAA.

\section{Inter-area Case:}

If the CBB detects that the destination address of a request is outside its controlled area, the operation proceeds as follows:

3. Since the destination is beyond the control of CBB, CBB forwards the RAR to the PBB.

4. When PBB receives the RAR, it refers to QPC for an entry with the source field equals to $s$ and the destination field equals to $d$. If the entry is found, the QoS path of the entry is retrieved. Then based on the LSDB, the PBB checks whether each link constituting the path is able to satisfy the request.

5. If the path retrieved satisfies the request, $\mathrm{PBB}$ retrieves the path information without re-calculating a new one. For the case in which neither such entry exists in the QPC nor the path found in the QPC supports the request, $\mathrm{PBB}$ performs the on-demand Dijkstra algorithm to calculate a feasible path for the request.

6. When the feasible path for the request is found, PBB updates the path information in the QPC (by adding a new entry or replacing the current one with new path information). At the same time, PBB updates its LSDB. For each link of the feasible path, bw is deducted from its available bandwidth to reflect the resource allocation to the request. Later, PBB sends an update link state message to the CBB of source area and $\mathrm{CBB}$ of the destination area to inform them to update their LSDB for the corresponding links.

7. Next, PBB generates a Resource Allocation Answer (RAA) and sends it to the requesting CBB. The RAA indicates whether the request is admitted or rejected. For a positive reply, the path information is inserted into the RAA.

8. When the source CBB receives the RAA from PBB, it forwards the RAA to the requesting router.

As soon as the QoS flow terminates, the router that has requested the QoS path sends a tear-down message to CBB. The CBB then updates the link QoS state information in the LSDB to reflect the termination of the flow. CBB also sends an update link state message to PBB to inform PBB to update its LSDB. QoS runs on TCP as the transport protocol for reliable transmission of route requests/replies, link QoS state update, and tear-down messages. 


\subsection{MPLS Path Setup}

Upon receiving the RAA from the local CBB, the source router checks whether the reply is positive or negative. If the reply is positive, the source router starts to setup an explicit Label Switched Path (LSP) along the path embedded in the RAA using Constraint-based Routing Label Distribution Protocol (CR-LDP). Constraint-based Routing (CR) takes into account both the hop-count and available bandwidth. Using CR, through QOSPF, selecting a path with higher hop-count but less loaded is possible. The IETF has defined a CR-LDP component to facilitate constraint-based routes in conjunction with MPLS. LDP is implemented using downstream-on-demand label distribution, and conservative label retention mode to exchange label binding between the peers. LDP is use to distribute labels in an MPLS environment based on the underlying routing protocol, that is, QOSPF.

The setting up of explicit LSP is explained as follows:

In the beginning, the initiating router sends a Label Request Message to its next hop. The next hop was specified in the explicit path embedded in the request. When the next hop receives the label request message, it further forwards the message to its next hop. The message is forwarded hop-by-hop until it reaches to the destination (the downstream). The downstream router then initiates the distribution of labels and the label/FEC binding. TCP as a reliable transport protocol is used for this signaling protocol. Upon receiving the request, the destination node binds a label to the request and distributes the label mapping message to its upstream. When a router receives the label mapping message, it extracts the label information, updates its Forwarding Information Base (FIB) tables and then binds another label to its upstream. The process continues until the label-mapping message reaches the source router. After the source router processes the message, the entire LSP path is considered set up successfully and the data transmission begins.

Table 4: CBR source characteristics

\begin{tabular}{|l|l|}
\hline Bit rate & $0.4 \mathrm{Mbps}$ \\
\hline Number of bits to be sent & $2 \mathrm{Mbits}$ \\
\hline Start time & $1 \mathrm{~s}$ \\
\hline Delay between call & $3 \mathrm{~s}$ \\
\hline Class of service & $\mathrm{EF}$ \\
\hline
\end{tabular}

\subsection{SIMULATION ENVIRONMENT AND RESULTS}

This section presents the simulation environment, results, and analysis of the performance of the proposed QoSHBB. Fig. 2 depicts the topology used in the simulation. The topology is derived from the MCI Internet backbone, which is an actual continent-wide topology used in other works [10,14]. Additional links and nodes are added to the MCI Internet backbone and the topology is partitioned into several areas. The modified MCI topology consists of 4 areas, 21 nodes, and 36 links with 3 levels of capacity. The bandwidths are scaled down from the actual values in order to reduce simulation overhead. The relative capacity among different links is adjusted to a smaller range. The resulting bandwidths for low, medium, and high capacity links are 4 Mbps, 5 Mbps, and 7 Mbps respectively.

In the topology, each router is linked to a customer site. Every site is connected to 12 applications, which generate the network traffic. The EF class of service was simulated using constant bit rate (CBR) source. Table 4 shows the parameters of the CBR source. Note that the start time is set to one (1) second in order to make sure that OSPF routing protocol converges prior to data transmission.

The simulation compared QoS-HBB with OPSF. For both the QoS-HBB and OSPF mechanisms, a total of 10 simulation sessions were performed, each with increasing traffic load. The simulation started with a normalized load of 1 , whose traffic rate and traffic size are shown in Table 4 . Then, in each subsequence run, the traffic rate was increased by $10 \%$. The simulation time for each session was set to 180 seconds, which is equivalent to $1.8 \times 10^{10}$ ticks in simulation time. 


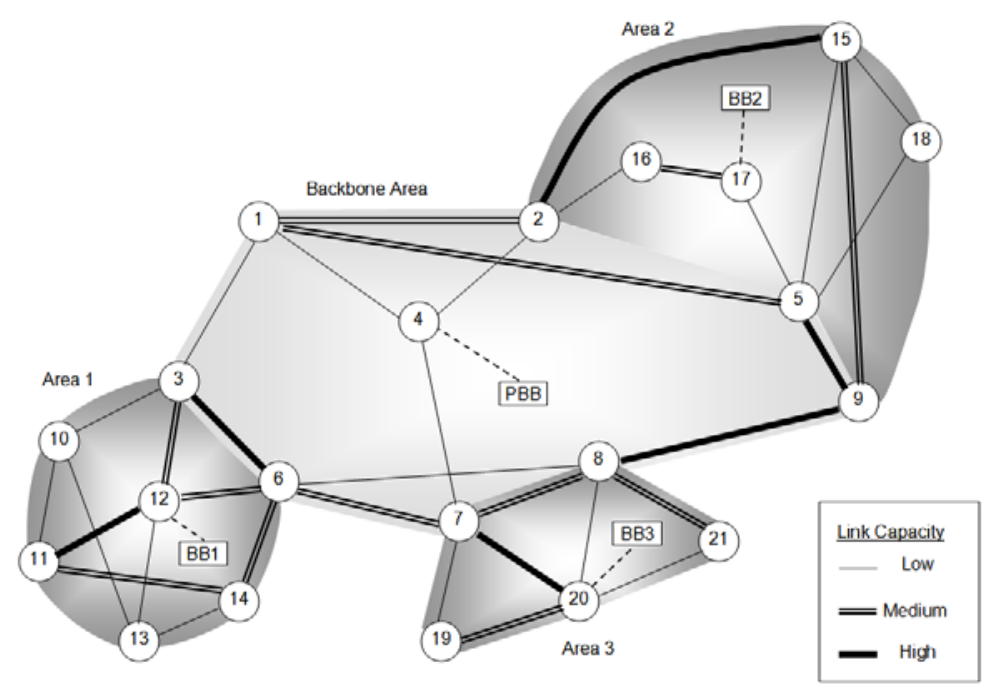

Fig. 2: Simulation Topology

\subsection{Packet Loss Ratio}

The packet loss ratio of a router is defined as follows:

$$
\text { packet loss ratio }=\quad \frac{\text { total number of packets dropped }}{\text { total number of packets received }} \times 100 \%
$$

The packet loss ratio for the entire network is calculated as a weighted average packet loss ratio among all routers, in which the weight is the total number of packets received at each router. This parameter shows the throughput of the routing scheme. A lower packet loss ratio indicates a better throughput. The result is shown in Fig. 3.

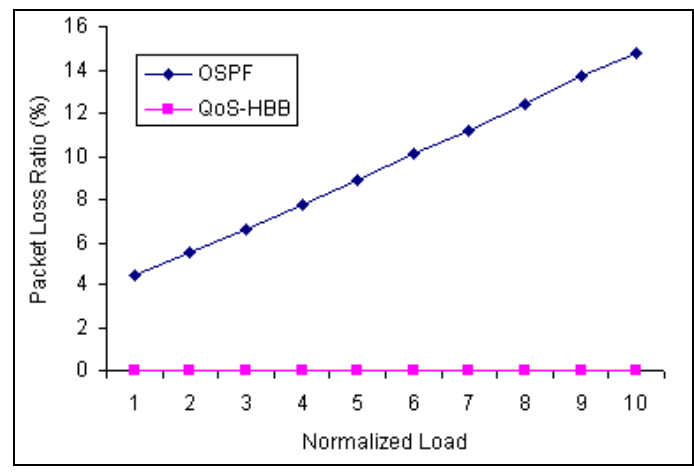

Fig. 3: Packet Loss Ratio

Fig. 3 shows that the packet loss ratio of OSPF increases linearly according to the traffic load. This result is anticipated, because in OSPF as there is no call admission control, and all traffic is admitted without regards of the congestion level of the network. As the network becomes increasingly saturated, more packets are dropped. On the other hand, the packet loss ratio for QoS-HBB is always equal to zero regardless the size of the traffic load. This is due to the call admission mechanism in QoS-HBB whereby the availability of resources is ensured before a call is admitted. 


\subsection{Link Utilization}

Link utilization is a common performance evaluation metric used to reflect the network resource utilization. Two types of link utilization were measured in this work: average link utilization and peak link utilization. Average link utilization gives an indication of the network throughput. For similar traffic load, higher average link utilization generally implies higher throughput. On the other hand, the peak link utilization reflects the load distribution among the network links. Lower peak utilization implies that the load is more evenly distributed in the network to support more traffic.

The link utilization is measured based on the long-term link utilization calculated at each outgoing interface of every router according to the following formula.

long-term link utilization $=$

total packets is scheduled

total simulation time $x$ link capacity

By considering long-term link utilization for all router interfaces in the network, the average link utilization is calculated as follows:

$$
\text { average link utilizatio } n=\frac{\sum_{i=1}^{n} \text { longterm link utilizatio } n \text { of interface } i \times \text { capacity of link } i}{\sum_{i=1}^{n} \text { capacity of link } i}
$$

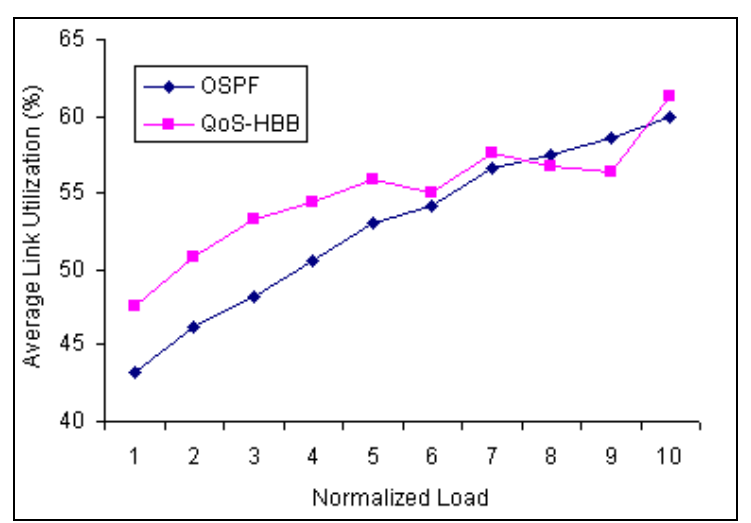

Fig. 4: Average Link Utilization

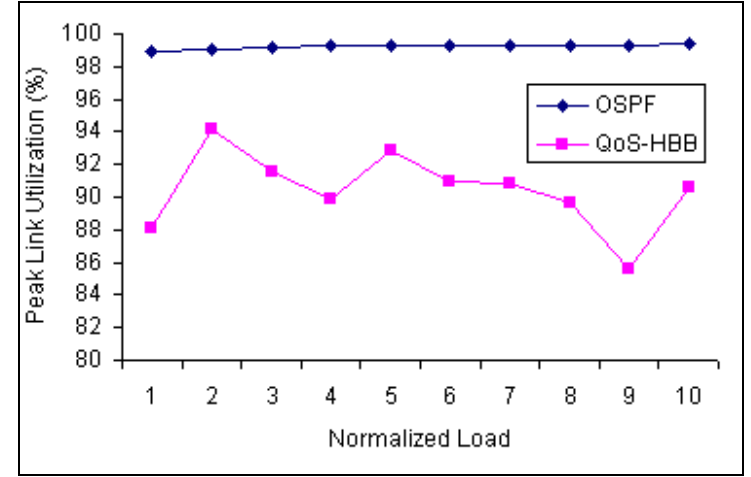

Fig. 5: Peak Link Utilization

The peak link utilization is the highest long-term link utilization measured among all the router interfaces. Fig. 4 and Fig. 5 show the results of the average and the peak link utilization. From these two figures, QoS-HBB has a higher average link utilization compared to OSPF due to the QoS provisioning of the bandwidth broker and the use of both hop-count and available bandwidth metrics in the paths selection process. In the case of peak link utilization, QoS-HBB outperforms OSPF by reducing the peak link utilization from 4.9 percent to 13.8 percent compared to OSPF. The lower value of peak link utilization proves that QoS-HBB is capable of distributing the traffic to less congested paths and hence avoiding the possibility of overusing certain links. In Fig. 5, since only the highest value (the peak) of QoS-HBB is recorded in each run, this value varies randomly between 85\% and 95\% and is independent of the traffic load. On the other hand, as indicated in Fig 4, when the average link utilization, instead of the peak value (single value) is recorded, as expected, this value increases proportionally to the traffic load. 


\subsection{End-to-End Delay}

The end-to-end delay of a packet is the interval between the time the packet is sent out at the source and the time the packet arrives at the destination. This parameter is an aggregate of queuing delay and propagation delay. During the simulation, the average and peak end-to-end delay was measured. The simulation results are shown in Fig. 6 and Fig. 7.

From Fig.6 and Fig. 7, QoS-HBB has a lower end-to-end delay compared to OSPF. By applying QoS-HBB, there is a drop of $95 \%$ to $98 \%$ in average end-to-end delay. For peak end-to-end delay, the gap between OSPF and QoSHBB becomes even larger. The peak end-to-end delay for OSPF is more than 200ms, whereas for QoS-HBB it is only around $5 \mathrm{~ms}$ which is a difference of about $97 \%$. The high end-to-end delay of OSPF scheme is mainly caused by queuing delay. This result verifies that in a congested network, the queuing delay has a much greater effect on the end-to-end delay than the propagation delay. The QoS-HBB scheme helps minimize network congestion and therefore in turn reduce the delay caused by queuing.

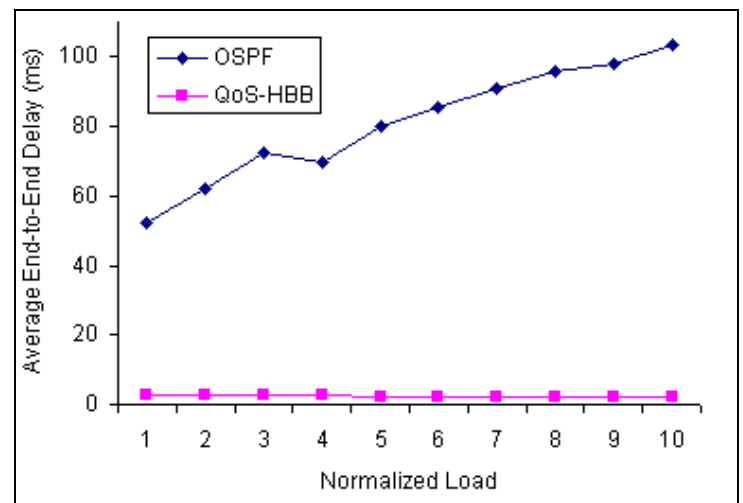

Fig. 6: Average End-to-End Delay

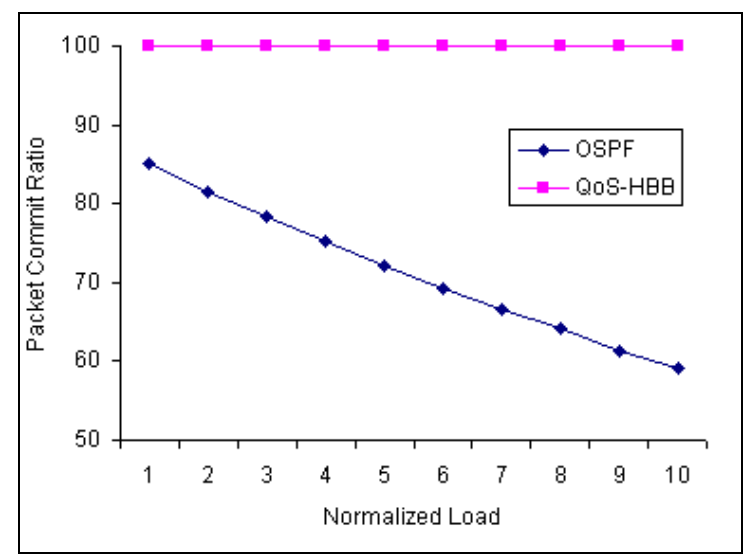

Fig. 8: Packet Commit Percent

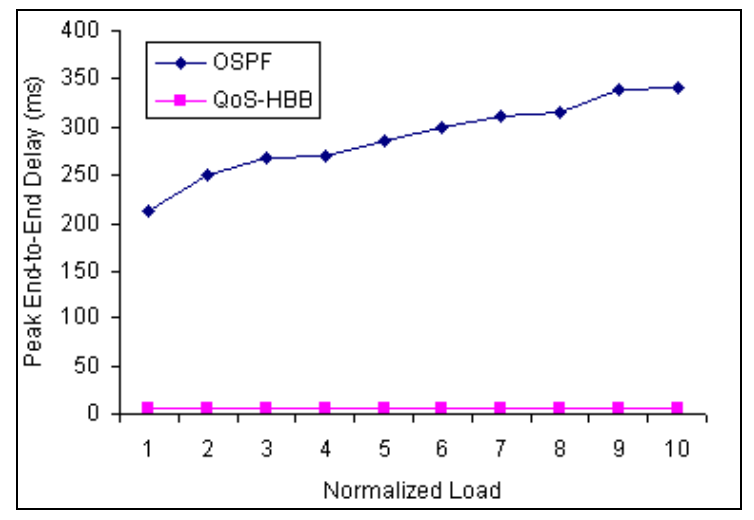

Fig. 7: Peak End-to-End Delay

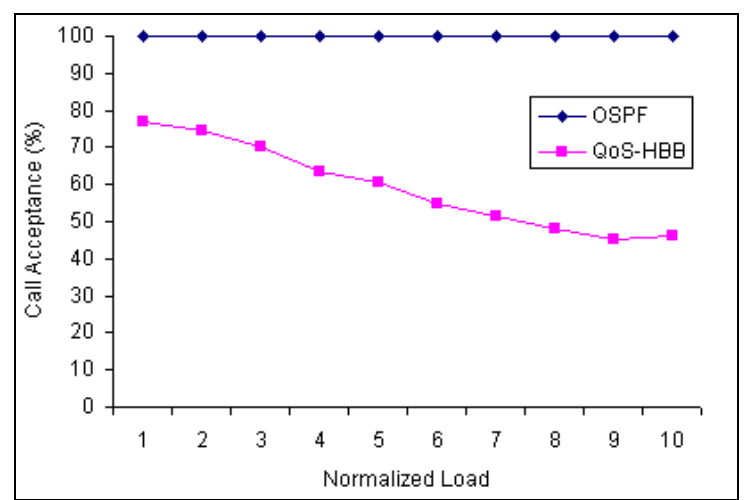

Fig. 9: Call Acceptance Ratio

\subsection{Packet Commit Percent}

The packet commit percent is an approximate indication about the per source packet loss ratio. This metric measures the percentage of packets that have been successfully sent from source to destination at the time of the measurement. The parameter is defined as follows. 
The result in Fig. 8 shows that the throughput of QoS-HBB is almost 100\% (i.e. 99.999\%). The loss of $0.001 \%$ is due to the fact that when the simulation ends, there are still packets in transit and these packets are yet to arrive at the destination. On the other hand, the performance of OSPF is poor with the packet commit percentage of only $85 \%$ at the beginning. The situation deteriorates when traffic becomes heavier and the packet commits percentage plunges below $60 \%$.

\subsection{Call Acceptance Ratio}

The call acceptance ratio is the ratio of the number of admitted request over total number of request made. As demonstrated in Fig. 9, the call acceptance ratio for OSPF is always 100\% as OSPF does not provide call admission control and thus there is no call blocking. On the other hand, for QoS-HBB, PBB and CBBs enforce admission control as well as QoS control. A call request will be rejected if there is no path in the network that can satisfy the request. The graph shows that the call acceptance ratio for QoS-HBB drops when traffic load increases. This is because as the traffic load increases, the network becomes more congested and the possibility of getting a feasible path becomes slimmer.

\subsection{Call Waiting Time}

As mentioned earlier, there is no admission control or resource reservation available for OSPF. Whenever a packet arrives, the packet is forwarded immediately by source based on the OSPF routing table. The proposed QoS-HBB, unlike OSPF, needs to go through several steps (mentioned in Section 3.6) before the source (the edge router) decides whether to accept a request. Hence, the purpose of measuring this parameter is to determine whether the time taken by QoS-HBB to accept or to reject a request is reasonable. The call waiting time is calculated as the interval between the time a request is sent and the time reply is received. The results for this parameter are shown in Fig. 10.

Fig. 10 shows that the call waiting time for the OSPF scheme is always zero because the packets are sent directly without the need to go through any admission control. On the other hand, the call waiting time for QoS-HBB is in the range of 4 to 7 milliseconds. It is always an overhead when admission control and routing decision are used. However, the short processing time in Fig. 10 proves that the impact of the call waiting time to the performance capability of QoS-HBB is negligible.

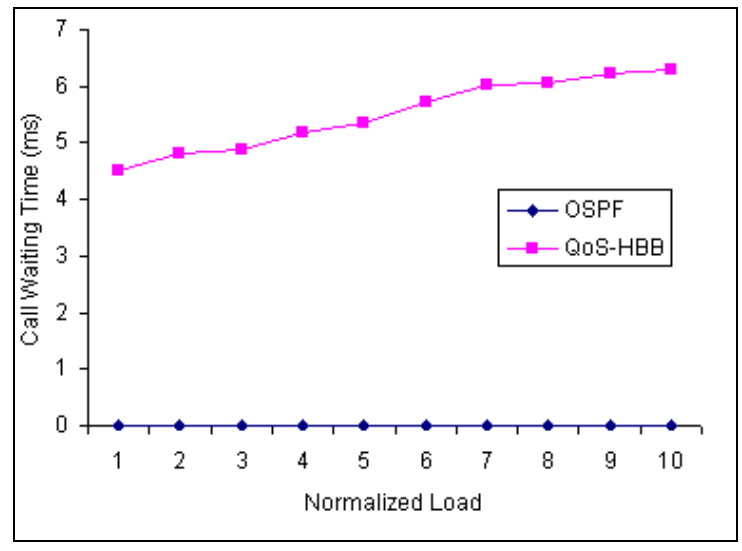

Fig. 10: Call Waiting Time

Overall, the simulation results show that the proposed QoS-HBB scheme significantly outperforms OSPF. QoSHBB has achieved an improvement with no packet loss and minimum end-to-end delay for all admitted requests. The benefits of QoS-HBB come with additional costs of call waiting time. Simulation results verify that this cost is insignificant. 


\subsection{CONCLUSIONS AND FUTURE WORK}

In this paper, an intra-domain hierarchical bandwidth broker architecture, namely QoS-HBB, is proposed. We have discussed how multiple bandwidth brokers can be designed and deployed within a single AS containing multiple areas. In QoS-HBB, routers are relieved from any QoS control and resource management as these jobs are delegated to CBBs and PBB. The two-level hierarchical bandwidth broker architecture is designed such that the burden of PBB is distributed to the CBBs. Without the hierarchical design, PBB would have end up as a bottleneck in the network. This is particularly desirable in a large network with high volume of traffic. Furthermore, in this design, the cost of on-demand path calculation in CBB and PBB is reduced using path caching scheme. The MPLS technique is incorporated in the proposal to speed up packet forwarding using MPLS label. The effectiveness of the proposed scheme over the original OSPF was demonstrated through simulation where significant improvements in packet loss ratio, link utilization, end-to-end delay, and throughput were observed.

QoS-HBB is only an intra-domain protocol defined partially for Bandwidth Broker-to-Bandwidth Broker (BB-to$\mathrm{BB})$ communication. Future study involves the study of how QoS-HBB can be extended to support inter domain BB-to-BB communication. Security and robustness are some of the issues in the development of bandwidth brokers. To improve security, robustness, and fault tolerance of the proposed bandwidth broker, enhanced security measures and backup bandwidth brokers should also be considered.

\section{REFERENCES}

[1] R. Braden, D. Clark, and S. Shenker, “Integrated Services in the Internet Architecture: an Overview”, RFC 1633, June 1994.

[2] S. Blake et al , “An Architecture for Differentiated Services”, RFC 2475, Dec.1998

[3] Chan Siew Yin et al, "Hop-by-hop QoS Routing using Statistical Distribution-free Approach”, Malaysian Journal of Computer Science, Vol 18, No 2, 2005.

[4] S. H. Lim et al, “Traffic Engineering Enhancement to QoS-OSPF in DiffServ and MPLS Networks”, IEE Proceedings-Communications, Vol. 151, No 1, February 2004.

[5] X. Xiao \& L. M. Ni, “Internet QoS: A Big Picture”, IEEE Network Magazine, March/ April 1999, pp. 8-18.

[6] S. Chen, \& K. Nahrstedt, “An Overview of Quality of Service Routing for Next-Generation High Speed Networks: Problems and Solutions," IEEE Network Magazine, Special Issue on Transmission and Distribution of Digital Video, Vol. 12 No. 6, 1998, pp. 64-79.

[7] D. L. Zhang; D. Ionescu, "QoS Performance Analysis in Deployment of DiffServ-Aware MPLS Traffic Engineering”, Eighth ACIS International Conference, Vol. 3, 2007, pp. 963 - 967.

[8] E.Crawley, R.Nair, B. Rajagopalan, \& H. Sandick, “A Framework for QoS-based Routing in the Internet”, RFC 2386, August 1998.

[9] D. Gosh, V. Sarangan, \& R. Acharja, “Quality of Service in IP Networks”, IEEE Transactions on Multimedia, Vol. 3, No. 2, June 2001, pp. 200-208.

[10] G. Apostolopoulos, R. Guerin, S. Kamat, \& S. K. Tripathi, "Quality of Service Based Routing: A Performance Perspective”, ACM SIGCOMM'98, Vancouver, B.C., 1998, pp. 17-28.

[11] Q. Ma \& P. Steenkiste, “Quality of Service Routing for Traffic with Performance Guarantees,” in Proceedings of IWQoS'97, Columbia Univ., New York, NY, May 1997.

[12] Z. Wang, \& J. Crowcroft, “Quality of Service Routing for Supporting Multimedia Applications”, IEEE Journal Selected Areas in Communications, Vol. 14 No. 7, 1996, pp. 1228-1234

[13] J. Moy, “OSPF Version 2”, RFC 2328, April 1998.

[14] G. Apostolopoulos, R. Guerin, \& S. Kamat, "Implementation and Performance Measurements of QoS Routing Extensions to OSPF”, in Proceedings of INFOCOM '99, New York, March 1999.

[15] K. Nichols, V. Jacobson, \& L. Zhang, “A Two-Bit Differentiated Services Architecture for the Internet”, RFC 2638, July 1999.

[16] Zhang et al., “On Scalable Design of Bandwidth Brokers”, IEICE Trans. Commun, Vol. E84-B No. 8, 
August 2001

[17] S. Gadgil, B. Dasarathy, F. Porter, K. Parmeswaran, \& R. Vaidyanathan, "Fast Recovery and QoS Assurance in the Presence of Network Faults for Mission-Critical Applications in Hostile Environments", in $13^{\text {th }}$ IEEE International Conference on Embedded and Real-Time Computing Systems and Applications. Aug. 2007, pp. $283-292$.

[18] S.S. Lee, S. Das, H. Yu, K. Yamada, G. Pau, \& M. Gerla, "Practical QoS network system with fault tolerance”, Computer Communications 26, 2003, pp. 1764-1774.

[19] D. A. Vivanco \& A. P. Jayasumana, "Bandwidth Brokering and Dynamic Resource Allocation in DiffServ Domains for Heterogeneous Applications”, in Proceedings of 27th IEEE Conference on Local Computer Networks, Tampa, FL, Nov. 2002, pp. 372-381.

[20] D Spence. Multidomain Bandwidth Broker Model. http://qbone.internet2.edu/bb/bboutline2.html. 1999, Accessed April, 2008.

[21] C. Bouras \& D. Primpas, "Path Finding Architectures for Interdomain Bandwidth Broker Operation”, in 14th IEEE International Conference on Network, Vol. 1, Sept. 2006, pp. 1 - 6.

[22] A. Terzis, L. Wang, J. Ogawa, \& L. Zhang, “A Two-Tier Resource Management Model for the Internet”. Global Telecomunications Conference (GLOBECOM '99), Vol. 3, pp. 1779-1791, December 1999.

[23] R. A. Guerin, A. Orda \& D. Williams, "QoS Routing Mechanisms and OSPF Extensions", in Proceedings of IEEE GLOBECOM, 1997, pp. 1903-1908.

\section{BIOGRAPHY}

Ling Teck Chaw obtained his PhD in 2005 from University of Malaya, Malaysia. He is an Associate Professor at Faculty of Computer Science \& Information Technology, University of Malaya. His research areas include core network research, inter-domain Quality of Service (QoS), Voice over IP (VoIP), grid computing, and network security.

K. K. Phang is an Associate Professor at the Faculty of Computer Science \& Information Technology, University of Malaya, Malaysia. He obtained his PhD in 2004 from University of Malaya. His current research interests include high speed computer network, network QoS, grid computing, traffic conditioning and traffic engineering, and fuzzy logic.

Ang Tan Fong obtained his M. Comp. Sc. in 2001 from University of Malaya. He is a lecturer at the Faculty of Computer Science \& Information Technology, University of Malaya, Malaysia. His research areas include web services, Voice over IP (VoIP) and grid computing.

Lim Gek Pei obtained her M. Comp. Sc. in 2005 from University of Malaya. She is currently a software engineer. 